10 Dunn DT, Newell ML, Mayaux, Kind C, Hutto C, Goedert JJ, et al. Mode of delivery and vertical transmission of HIV-1: a review of prospective studies. Perinatal AIDS collaborative transmission studies. J AIDS Hum Retrovirol 1994;7:1064-6.

11 Sculpher MJ, Gibb D, Ades AE, Ratcliffe J, Duong T. Modelling the costs of paediatric HIV infection and AIDS: comparison of infected children of paediatric HIV infection and AIDS: comparison of infected child
born to screened and unscreened mothers. AIDS 1998;12:1371-80.

12 Biggar RJ, Miotti PG, Taha, Mtimavalye L, Broadhead R, Justesen A, et al. Perinatal intervention trial in Africa: effect of birth canal cleansing intervention to prevent HIV transmission. Lancet 1996;347:1647-50.

13 Semba RD, Miotti PG, Chipangwi J, Saah AJ, Canner JK, Dallabetta GA, et al. Maternal Vitamin A deficiency and mother-to-child transmission of HIV-1. Lancet 1994:343:1593-7.

14 UNAIDS and World Health Organisation. Guidance modules on anti-retroviral treatments-module 6. The use of antiretroviral drugs to reduce mother to child transmission of HIV. Geneva: UNAIDS/WHO, 1998.

15 Marseilles E, Kahn JG, Saba J. Cost-effectiveness of anti-retroviral drug therapy to reduce mother-to-child HIV transmission in sub-Saharan Africa. AIDS 1998;12:939-48.

16 Mansergh G, Haddix AC, Steketee RW, Nieburg PI, Hu DJ, Simonds RJ, et al. Cost-effectiveness of short-course zidovudine to prevent perinatal HIV type 1 infection in a sub-Saharan African developing country setting. JAMA 1996;276:139-45.

17 Wilkinson D, Floyd K, Gilks CF. Antiretroviral drugs as a public health intervention for pregnant women in rural South Africa: an issue of costeffectiveness and capacity. AIDS 1998;12:1675-82.

18 Centres for Disease Control and Prevention. Administration of zidovudine during late pregnancy and delivery to prevent perinatal HIV zidovudine during late pregnancy and delive 1998:47:151-4.

19 Department of Health and Population Development, South Africa. Seventh national HIV survey of women attending antenatal clinics of the public health services in the Republic of South Africa, October/ November 1996. Epidemiological Comments 1996;23:4-16.

20 Harrison D, Nielson M, ed. South African health review-1995. Durban: Health Systems Trust and the Henry J Kaiser Foundation, 1995.

21 World Bank. World development report 1993-investing in health. New York: Oxford University Press, 1993.

22 Wagstaff L, de Wet T, Anderson A. Infant feeding: birth to ten. Urbanisation and Health Newsletter 1993;18:9-16.
23 Ross SM, van Middelkoop A, Khoza NC. Breast feeding practices in a black community. S Afr Med J 1983;63:23-5.

24 Central Statistical Service. Statistics in brief-1997. Pretoria: Central Statistical Service, 1997.

25 Cunningham AS, Jelliffe DB, Patrice Jelliffe EF. Breast-feeding and health in the 1980s: a global epidemiologic review. J Pediatr 1991;118:659-66.

26 Kinghorn AW. Projections of the costs of anti-retroviral interventions to reduce mother-to-child transmission of HIV in the South African public sector. Johannesburg: HIV Management Services Technical Report, 1998.

27 Zwi KJ, Pettifor JM, Söderlund RN. Paediatric hospital admissions at a South African urban regional hospital: the impact of HIV 1992-1997. Ann Trop Paed (in press).

28 Söderlund N. Likely costs of hospital care benefits to be covered by a Social Health Insurance fund-a report to the National Department of Health. Johannesburg: Centre for Health Policy, 1997.

29 Söderlund N, Peprah E. An essential hospital package for South Africa-selection criteria, costs and affordability. Johannesburg: Centre for Health Policy, 1998. (Monograph No 52.)

30 Brown M, van den Heever A. Report by the consultants on the existing expenditure trends and functional costs to support the function analysis and rationalisation programme in terms of the National Health Plan and the Reconstruction and Deudopment Prograne. Johannesburg: PWV Province struction and Development Programme. Joha

31 Regional Health Management Information System (ReHMIS). Pretoria: National Department of Health of South Africa, 1995.

32 Briggs A, Sculpher M. An introduction to Markov modelling for economic evaluation. Pharmacoeconomics 1998;13:397-409.

33 Tess BH, Rodrigues LC, Newell ML, Dunn DT, Lago TD. Infant feeding and risk of mother-to-child transmission of HIV in Sao Paulo State, Brazil. Sao Paulo collaborative study for vertical transmission of HIV-1. J AIDS Hum Retrovirol 1998; 19:189-94.

34 Bertolli J, St Louis ME, Simonds RJ, Nieburg P, Kamenga M, Brown C, et al. Estimating the timing of mother-to-child transmission of human immunodeficiency virus in a breastfeeding population in Kinshasa, Zaire. J Infect Dis 1996;174:722-6.

35 UNAIDS and World Health Organisation. Guidance modules on antiretroviral treatments-module 2. Introducing antiretroviral treatments into health systems: economic considerations. Geneva: UNAIDS/WHO, 1998.

(Accepted 30 March 1999)

\title{
Universal HIV screening of pregnant women in England: cost effectiveness analysis
}

\author{
M J Postma, E J Beck, S Mandalia, L Sherr, M D S Walters, H Houweling, J C Jager
}

Papers pp 1650,

\section{0}

National Institute of Public Health and the Environment, PO Box 1,3720 BA Bilthoven,

Netherlands

Department of Health Services Research

M J Postma, econometrician J C Jager, biomathematician

Department of Infectious Diseases Epidemiology

H Houweling, epidemiologist continued over

BMJ 1999;318:1656-60

\begin{abstract}
Objective To estimate the cost effectiveness of universal, voluntary HIV screening of pregnant women in England.

Design Cost effectiveness analysis. Cost estimates of caring for HIV positive children were based on the stage of HIV infection and calculated using data obtained from a London hospital between 1986 and 1996. These were combined with estimates of the health benefits and costs of antenatal screening so that the cost effectiveness of universal, voluntary antenatal screening for HIV infection in England could be estimated.

Main outcome measures Lifetime, direct costs of medical care of childhood HIV infection; life years gained as a result of the screening programme; net cost per life year gained for different pretest counselling costs; and different prevalence rates of pregnant women who were unaware that they were HIV positive.
\end{abstract}

Results Estimated direct lifetime medical and social care costs of childhood HIV infection were $£ 178300$ using a 5\% discount rate for time preference (1995-6 prices). In high prevalence areas screening pregnant women for HIV is estimated to be a cost effective intervention with a net cost of less than $£ 4000$ for each life year gained. For areas with comparatively low prevalence rates, cost effectiveness could be less than $£ 20000$ per life year gained, depending on the number of pregnant women who are unaware that they are infected and local screening costs.

Conclusions Our results confirm recent recommendations that universal, voluntary antenatal HIV screening should be implemented in the London area. Serious consideration of the policy should be given for other areas in England depending on local prevalence and screening costs.

\section{Introduction}

In England the uptake of antenatal HIV screening is comparatively low despite the existence of guidelines on antenatal screening for pregnant women. ${ }^{1}$ Detection of HIV infection in pregnant women allows the risk of mother to child transmission to be reduced..$^{2-4}$ This study analyses the cost effectiveness of an antenatal HIV screening programme.

\section{Methods}

We assessed the cost effectiveness of universal, voluntary HIV screening of pregnant women in England in terms of healthcare costs to the NHS. A staged, progression of disease model was developed 


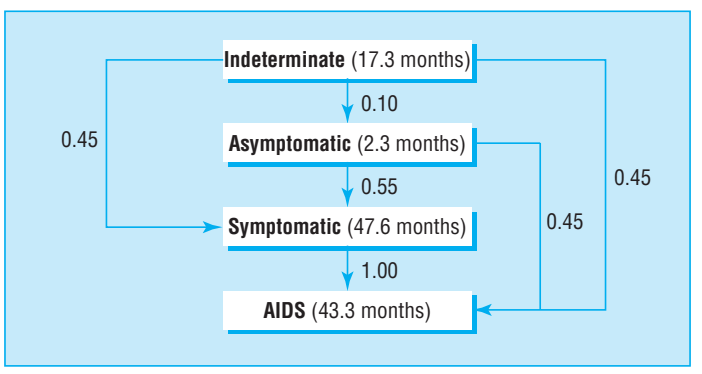

Fig 1 Model shows the progression of disease in HIV positive children. The mean duration of stay in each of the stages of the disease and the probability distribution for progression from one stage to another are also shown

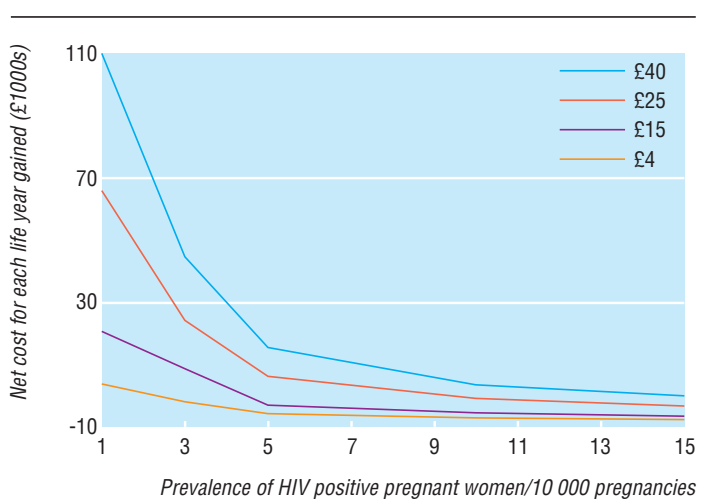

Fig 2 Cost for each life year gained with universal antenatal screening for different prevalences of women who do not know that they are HIV positive at different screening costs

using clinical and epidemiological data and estimates of the cost of caring for children with HIV calculated between 1986 and $1996 .^{5-6}$ The analysis is based on the effects of testing one pregnant woman who is unaware that she is infected with HIV, and we excluded from the analysis women who request an HIV test during pregnancy. Effectiveness was measured in terms of life years gained among children in whom infection with HIV was averted and in life years gained because of earlier antiretroviral treatment of the mothers. Costs considered included those of serological screening for HIV antibodies, pretest and post-test discussions and counselling, antiretroviral treatment, elective caesarean section, and the additional cost of formula feeding compared with breast feeding.

The probabilities for mother to child transmission used within the model were $14 \%$ for breast feeding, ${ }^{2}$ $18 \%$ during pregnancy and vaginal delivery in the absence of zidovudine treatment, ${ }^{3} \quad 10 \%$ during pregnancy and caesarean delivery without zidovudine, ${ }^{3}$ $8 \%$ with zidovudine treatment for the mother during pregnancy and for the child with a vaginal delivery, ${ }^{4}$ and a $6 \%$ transmission rate was assumed for zidovudine treatment and a caesarean delivery. Independent probabilities were assumed for breast feeding and delivery. The zidovudine regimen used in the model was similar to AIDS clinical trials group protocol $076 .{ }^{4}$ In the absence of preventive measures the probability of mother to child transmission was calculated to be $29 \%$, which decreased to $23 \%$ if a caesarean section was performed, and to $6 \%$ if all preventive measures were implemented. ${ }^{2-4}$ Four clinical stages of
HIV infection were considered: indeterminate, asymptomatic, symptomatic non-AIDS, and AIDS. ${ }^{7}$ The duration of stay within each clinical stage was assumed to be exponentially distributed (fig 1). Transition rates between the stages are described in figure 1 .

The lifetime costs of hospital and community care for a child infected with HIV were estimated at $£ 178300$ for the 1993-4 financial year indexed to $1995-6$ prices $^{8}$ and were discounted at $5 \%$ (3\% and 7\% in the sensitivity analyses). ${ }^{9}$ Lifetime costs of caring for a child infected with HIV were varied in the sensitivity analysis.

We assumed that the uptake of zidovudine among HIV positive pregnant women was $75 \%$ and that elective caesarean sections occurred in $40 \%$ of deliveries in HIV positive women. ${ }^{10-11}$ The rate of emergency caesarean sections among HIV positive women was assumed to be similar to that of the general population, namely $15 \%$ of deliveries. Among women known to be HIV positive, we assumed that $95 \%$ would refrain from breast feeding compared with $23 \%$ in the general population. ${ }^{10-11}$ In the sensitivity analysis lower and higher uptakes of these interventions were considered

The cost of 20 weeks' treatment with zidovudine (14 weeks for the mother and 6 for the child) is $£ 600$ (\$960), 91\% of which is for zidovudine taken during pregnancy, $8 \%$ for zidovudine during delivery, and $1 \%$ for zidovudine given to the newborn. The cost of a vaginal delivery is $£ 400$; an elective caesarean costs $£ 1100$, and an emergency caesarean costs $£ 1300 .{ }^{12} \mathrm{In}$ this model the first test was an enzyme linked immunosorbent assay with 100\% sensitivity and 99.9\% specificity; it was followed by a set of confirmatory enzyme linked immunosorbent assays. The costs for these tests were estimated at $£ 4$ per woman (T Oliver, personal communication). False positive results from the first round were considered in the costing; false positive results after all tests were completed were not considered. The current costs of screening and pretest counselling were estimated at $£ 40 .{ }^{13}$ Costs below $£ 40$ reflect a situation in which universal screening for HIV is integrated into routine antenatal care. For illustrative purposes an evaluation at marginal costs-that is, test costs only-was included. The cost of counselling women who tested positive for HIV infection was set at $£ 50$. The additional cost of formula feeding over breast feeding was estimated to be $£ 800$ (table 1 ). ${ }^{14}$

Cost effectiveness was expressed as net cost per life year gained. The net cost comprised the total costs of antenatal screening minus the screening benefits of averted health care for children infected with HIV. Life years gained were calculated by comparing life years lost due to HIV infection with and without antenatal

Table 1 1995-6 prices (£) used in the cost effectiveness analysis of universal, voluntary antenatal HIV screening

\begin{tabular}{lr} 
Lifetime cost of care for child infected with HIV & $\mathbf{1 7 8} \mathbf{3 0 0}$ \\
\hline Zidovudine treatment of mother and newborn & 600 \\
\hline Vaginal delivery & 400 \\
\hline Elective caesarean delivery & 1100 \\
\hline Emergency caesarean delivery & 1300 \\
\hline Screening costs (test plus pretest counselling) & 40 \\
\hline Post-test counselling (only if HIV positive) & 50 \\
\hline Formula feeding & 800 \\
\hline Postpartum triple therapy for the mother per asymptomatic patient year gained & 12300 \\
\hline
\end{tabular}
School of Medicine (St Mary's Campus) London W2 1PG

Department of Epidemiology and Public Health EJ Beck, senior lecturer S Mandalia, statistics

Department of Paediatrics senior lecturer

Department of Primary Care and Population Science, Royal Free Hospita School of Medicine, London NW3 2PF

L Sherr,

senior lecturer

Correspondence to: Dr Beck

e.beck@ic.ac.uk
Imperial College research assistant in

M D S Walters, 
Table 2 Net cost $(£)$ per life year gained with the introduction of universal, voluntary antenatal screening for different prevalences of women who are unaware that they are HIV positive per 10000 pregnancies

\begin{tabular}{|c|c|c|c|c|}
\hline \multirow[b]{2}{*}{ Prevalence } & \multicolumn{4}{|c|}{ Model } \\
\hline & $1 / 10000$ & $1 / 10000$ & $15 / 10000$ & $15 / 10000$ \\
\hline Screening costs & $£ 40$ & $£ 4$ & $£ 40$ & $£ 4$ \\
\hline \multicolumn{5}{|l|}{ Scenario } \\
\hline Discount rate $3 \% \dagger$ & 76600 & 4400 & 1800 & Cost saving \\
\hline Discount rate $7 \% \dagger$ & 151600 & 10400 & 5200 & Cost saving \\
\hline Two life years gained for the mother & 90800 & 8300 & 5200 & Cost saving \\
\hline Low uptake of interventions§ & 138100 & 10800 & 6100 & Cost saving \\
\hline High uptake of interventionsf & 103800 & 5700 & 2100 & Cost saving \\
\hline Low lifetime cost of caring for child infected with HIV†† & 116600 & 9400 & 5500 & Cost saving \\
\hline 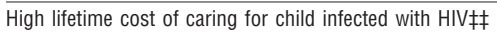 & 112200 & 5100 & 1100 & Cost saving \\
\hline
\end{tabular}

${ }^{*}$ Discounted at $5 \%$. Uptake of interventions in the reference case is $75 \%$ for treatment with zidovudine, $40 \%$ for elective caesarean section, and $95 \%$ for formula feeding. Lifetime cost of care for child infected with HIV in the reference case is $£ 178300$. †Compared with $5 \%$ in the reference case. $\neq$ Compared with one year in the reference case. §Low uptake scenario corresponds to $60 \%$ for treatment with zidovudine, $20 \%$ for elective caesarean section, and $80 \%$ for formula feeding. IHigh uptake scenario corresponds to $90 \%$ for treatment with zidovudine, $60 \%$ for elective caesarean section, and $100 \%$ for formula feeding. $\uparrow+75 \%$ of costs of reference case. $\$ \neq 125 \%$ of costs of reference case.

screening. Seventy life years lost is equal to the remaining life expectancy at age 7 ; this is the estimated average age of death for a child infected with HIV. Life years gained were discounted but as discounting health benefits is controversial, ${ }^{15}$ non-discounted life years gained are also presented. A gain of one year was assumed to reflect the health benefit for HIV positive mothers of starting antiretroviral treatment earlier. As most mothers would be asymptomatic, the costs of triple therapy for the mother with a protease inhibitor were included at $£ 12300$ per patient year $^{16}$; this combination therapy was used in the model as it has the highest cost. ${ }^{16}$ However, as the intermediate and long term benefits of triple therapy are unknown, cost estimates for long term treatment with triple therapy were not included. The assumptions of the impact of screening on the mother were investigated in the sensitivity analysis.

\section{Results}

\section{Reference case}

For the reference case the cost per life year gained varied from a situation of cost savings to a cost of $£ 114000$ (fig 2, table 2). If more than one woman in every 10000 pregnancies is unaware that she is HIV positive and the costs of screening are below $£ 40$, better cost effectiveness ratios are achieved. For example, if the prevalence of HIV among pregnant women is $10 / 10000$ pregnancies, the net cost for each life year gained is $£ 7300$ when screening costs $£ 40$; the net cost for each life year gained is $£ 10200$ when there is a prevalence of 5/10 000 pregnancies and screening costs $£ 25$. If screening costs fall further then situations of cost savings become possible. For example, if the screening costs are below $£ 8$ per woman at a prevalence of $5 / 10000$ pregnancies then screening becomes cost saving. If the prevalence is $15 / 10000$ pregnancies then universal antenatal screening costs $£ 3300$ per life year gained when screening costs $£ 40$.

In the reference case the lifetime cost of care for children infected with HIV for each mother detected is $£ 29100$ less than the costs accumulated in the absence of screening. Furthermore, the costs of treatment with zidovudine, counselling after testing, extra caesarean sections, and extra formula for feeding amount to $£ 1300$. At a prevalence of 15 women unaware that they are HIV positive per 10000 pregnancies, screening costs are $£ 26700$ ( $£ 40$ per case of screening) for detecting HIV infection in this one woman. Differences in the costs of the reference case were compared with a situation of no screening.

\section{Sensitivity analysis}

Relevant parameters were varied between low $(1 / 10000)$ and high prevalences $(15 / 10000)$ of women unaware that they were HIV positive and low and high screening costs ( $£ 4$ and $£ 40$ respectively). Major improvements in cost effectiveness are found when the discount rate is lowered to $3 \%$, when life years gained are not discounted, and when there is a high lifetime cost of paediatric HIV care (table 2). Assuming that two life years are gained instead of one for the mother results in an improvement in cost effectiveness only for the situation of low prevalence and high screening costs. In the other situations the costs of

Table 3 Break even costs of universal, voluntary antenatal HIV screening in 1995-6 prices at a prevalence of 14 women unaware that they are HIV positive per 10000 pregnancies under different scenarios

Scenario

Cost (£)

22

Discount rate $3 \% \dagger$

Discount rate $7 \% \dagger$

Life years gained, not discounted

No life years gained for the mother $\neq$

Two life years gained for the motherł

Low uptake of interventions§

High uptake of interventions

Low lifetime cost of caring for child infected with HIV††

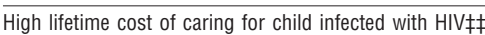

${ }^{*}$ Discounted at $5 \%$. Uptake of interventions in the reference case is $75 \%$ for treatment with zidovudine, $40 \%$ for elective caesarean section, and $95 \%$ for formula feeding. Lifetime cost of care for child infected with HIV in the reference case is $£ 178300$. †Compared with $5 \%$ in the reference case. $\ddagger$ Compared with one year in reference case. §Low uptake scenario corresponds to $60 \%$ for treatment with zidovudine, $20 \%$ for elective caesarean section, and $80 \%$ for formula feeding. IHigh uptake scenario corresponds to $90 \%$ for treatment with zidovudine, $60 \%$ for elective caesarean section, and $100 \%$ for formula feeding. $\dagger+75 \%$ of costs of reference case. $\neq \neq 125 \%$ of costs of reference case. 
treatment during the life years gained caused a lessening in cost effectiveness. If there are no life years gained for the mother whose infection is detected by the programme, then screening becomes cost saving at current screening costs and a prevalence of 15/10 000. This reflects the cost effectiveness of that part of the screening programme that focuses on reducing the transmission of infection from mother to child. The high prevalence combinations that were cost saving in the reference case remain cost saving. The sensitivity analysis shows that adequate uptake of interventions is crucial in achieving good cost effectiveness and that cost effectiveness is sensitive for the lifetime costs of caring for a child infected with HIV if the prevalence is high or test costs are low.

Finally, to illustrate how the cost per screening visit can be varied to break even, the sensitivity analysis was performed assuming a prevalence of 14 women unaware that they were infected with HIV per 10000 pregnancies, a prevalence recently reported for London. ${ }^{17}$ The break even point is the situation that occurs when screening costs equal the averted costs of caring for a child infected with HIV. In most situations, screening costs below $£ 25$ result in cost savings when the costs of averted health care outweigh the costs of screening and intervention (table 3). If universal HIV screening is integrated in to routine antenatal care then screening costs might drop well below the current $£ 40$.

\section{Discussion}

In situations in which the prevalence of pregnant women who are unaware that they are HIV positive is greater than $5 / 10000$ pregnancies and screening costs are $£ 40$ per test, screening for HIV infection might be a cost effective intervention with a net cost per life year gained of less than $£ 20000$. With a prevalence of $14 / 10000$ pregnancies universal antenatal screening would cost $£ 3900$ per discounted life year gained, $£ 1000$ per non-discounted life year gained, and would be cost saving at screening costs below $£ 22$. This compares to costs of about $£ 2500$ per non-discounted life year gained for both antenatal screening for hepatitis B and the NHS breast screening programme. ${ }^{18-19}$ Universal, voluntary antenatal HIV screening would be a cost effective intervention in London.

In areas of low prevalence where 1 pregnant woman per 10000 pregnancies is unaware of being infected with HIV and when screening costs are less than $£ 15$ the cost per life year gained is less than $£ 40000$. For regions with a prevalence of $3 / 10000$ pregnancies, screening costs of less than $£ 25$ bring the cost per life year gained to below $£ 20000$. Universal antenatal HIV screening seems to be cost effective for some regions outside London.

The cut off point at which the cost for each life year gained becomes acceptable differs over time and between societies. One Canadian article placed this point at $\$$ C20 000 per life year gained, ${ }^{20}$ whereas the point in the United States currently is around $\$$ US50 000 per life year gained..$^{21}$ No cut off point has yet been defined for England, although one has recently been defined in terms of seroprevalence. ${ }^{22}$

\section{Limitations of the model}

The costs of terminating a pregnancy for reasons related to HIV were not incorporated into the model.
Since the costs of terminating a pregnancy are below $£ 500$, their inclusion would make the cost effectiveness ratios more favourable.

It was assumed that all pregnant women offered the test would agree to be tested. If women who decline testing are at a higher risk of HIV infection than those who agree to be tested, cost effectiveness ratios may become less favourable. An increased uptake of screening would improve the cost effectiveness of such programmes and vice versa; however, early access to services, and barriers of language and literacy are all factors which may influence uptake.

Many important psychological factors cannot easily be costed and therefore were not incorporated in to the model. The cost of training additional healthcare professionals was also not included in the model. Additional training might be required especially if antenatal HIV screening with pretest discussions and counselling were offered outside of sexually transmitted disease clinics by comparatively inexperienced staff. These factors would not, however, dramatically change the study's general conclusions.

This model assumed that all women whose HIV infection was detected would be offered treatment with zidovudine and not combination therapy. Guidelines continue to recommend the use of zidovudine, ${ }^{23}$ although the minimum duration of treatment required to prevent transmission to the child is being investigated. ${ }^{24-25}$ However, if combination therapy were adopted during pregnancy (R Smith, personal communication, 1998), the impact on the model would be limited. Assuming costs of an additional $£ 2000$ for the use of a triple combination therapy regimen during pregnancy, the estimates of the cost effectiveness increase by less than $£ 1000$.

Neither the possible reduction in HIV transmission, due to behavioural change once a woman learns that she is infected, nor indirect costs were included in the model. A transmission rate of $6 \%$ was assumed if all interventions were implemented. This may be an overestimate; a recent study reported a transmission rate of only $1 \%$ if all interventions discussed were used.$^{26}$ All of these factors would produce more favourable cost effectiveness ratios if they had been incorporated. For example, if the $1 \%$ transmission probability were applicable to the London area, the cost per discounted life year gained would be reduced to $£ 2700$.

\section{Conclusion}

Since the incidence of mother to child transmission of HIV can successfully be reduced, the introduction of universal, voluntary antenatal screening needs to be considered. The availability of reliable cost estimates of treatment for HIV positive children enabled the first cost effectiveness assessment of antenatal screening for HIV in England to be performed. ${ }^{5-6}$ Although uncertainties remain and more detailed information is needed to further develop assessments of cost effectiveness, the results presented to date indicate that in areas of high prevalence, such as London, universal, voluntary antenatal screening of pregnant women is cost effective. For areas of lower prevalence the cost effectiveness of such screening programmes could be well below $£ 20000$ per life year gained; antenatal screening for HIV should be considered depending on possible reductions in screening costs and local preva- 
and cost of community service provision. London: Department of Epidemiology and Public Health, Imperial College School of Medicine at St Mary', 1997.

- The lifetime costs of care for a child infected with HIV have been estimated at $£ 178300$

- Screening pregnant women for HIV can avert this cost and lead to gains in life years for both mothers and children

- Universal, voluntary antenatal HIV screening is estimated to be a cost effective intervention with cost saving potential in areas in which there is a high prevalence of HIV infection among pregnant women

- In areas with lower prevalence rates, cost effectiveness could be well below $£ 20000$ per life year gained, and universal, voluntary antenatal screening could be considered

lence rates. These conclusions confirm the recent recommendations of the Intercollegiate Working Party for Enhancing Voluntary Confidential HIV Screening in Pregnancy. ${ }^{27}$

The authors thank Jeni Beecham, Margaret Brandeau, Miranda Mugford, Marie-Louise Newell, Angus Nicoll, Tony Oliver, Doug Owens, and Richard Smith, as well as our referees for their invaluable assistance at various stages of the study.

Contributors: MJP and EJB conceived the idea for the project. MJP developed the cost effectiveness model in conjunction with EJB, SM, LS, MDSW, HH, and JCJ. EJB and SM provided the cost data that were used in the model. LS provided particular input on the social context. MDSW provided specific input on the clinical context relevant to the analyses as well as providing clinical care to many of the children who participated in the original study. MJP and EJB were primarily responsible for writing the paper; the paper was written in conjunction with SM, LS, MDSW, HH, and JCJ. MJP and EJB are guarantors of the study.

Funding: European Union Concerted Action on Multinational AIDS Scenarios (contract No BMH1-CT94-1723).

Competing interests: None declared.

1 Mercy D. Antenatal HIV screening. BMJ 1998;316:241-2.

2 Dunn DT, Newell ML, Ades AE, Peckham CS. Risk of human immunodeficiency virus type 1 transmission through breastfeeding. Lancet 1992:340:585-8.

3 European collaborative study. Caesarean section and risk of vertical transmission of HIV-1 infection. Lancet 1994;343:1464-7.

4 Connor EM, Sperling RS, Gelber R, Kiselev P, Scott G, O'Sullivan MJ, et al. Reduction of maternal-infant transmission of human immunodeficiency virus type 1 with zidovudine treatment. $N$ Engl J Med 1994:331:1173-80.

5 Beck EJ, Mandalia S, Griffith R, Walters MDS, Levin M, Boulton M, et al. The hospital and community services study of families with HIV infection: initial analyses of hospital service provision and costs for HIV-infected children St Mary's Hospital, 1986-1994. London: Department of Epidemiology and Public Health, Imperial College School of Medicine at St Mary's, 1997.

6 Beck EJ, Griffith R, Mandalia S, Beecham J, Boulton M, Walters MDS, et al. The hospital and community services study of families with HIV infection: use
7 Centers for Disease Control. Classification system for human immunodeficiency virus (HIV) infection in children under 13 years of age. MMWR Morb Mortal Wkly Rep 1987;36:225-31.

8 NHS Executive. Hospital and community health services revenue (pay and prices) inflation index. Leeds: NHS Executive Finance and Performance Department A, 1997

9 Jefferson T, Demicheli V, Mugford M. Elementary economic evaluation in health care. London: BMJ Publishing Group, 1996.

10 Gibb DM, MacDonagh SE, Tookey PA, Duong T, Nicoll A, Goldberg D, et al. Uptake of interventions to reduce mother-to-child transmission of HIV in the United Kingdom and Ireland. AIDS 1997;11:F53-8.

11 Royal College of Obstetricians and Gynaecologists. National study of HIV in pregnancy. London: RCOG, 1997. (Newsletter 31.)

12 Mugford M. How does the method of cost estimation affect the assessment of costeffectiveness in health care [DPhil thesis]. Oxford: University of Oxford, 1996.

13 Chrystie IL, Zander L, Tilzey A, Wolfe CDA, Kenny A, Banatvala JE. Is HIV screening in pregnancy worthwhile: can we afford it? AIDS Care $1995 ; 7: 135-42$.

14 Savage-King F. Breast feeding programmes and HIV questions. Geneva: Marathon Multimedia, Cicero, 1998. [Abstract No 310.]

15 Parsonage M, Neuberger H. Discounting of health benefits. Health Economics 1992;1:71-6.

16 Beck EJ, Pozniak A, Molesworth A, Power A, Griffin J, Easterbrook P, et al. Changing cost of English HIV service provision, 1996-1997. Int J STD AIDS (In press).

17 Nicoll A, McGarrigle C, Brady ARG, Ades AE, Tookey P, Duong T, et al. Epidemiology and detection of HIV-1 among pregnant women in the United Kingdom: results from national surveillance. BMJ 1998; 316:253-8.

18 Jordan R, Law M. An appraisal of the efficacy and cost-effectiveness of antenatal screening for hepatitis B. J Med Screen 1997;3:117-27.

19 Boer R, de Koning H, Threlfall A, Warmerdam P, Street A, Friedman A, et al. Cost effectiveness of shortening screening interval or extending age range of NHS breast screening programme: computer simulation study. BMJ 1998;317:376-9.

20 Laupacis A, Feeny D, Detsky AS, Tugwell PX. How attractive does a new technology have to be to warrant adoption and utilization? Tentative guidelines for using clinical and economic evaluations. Can Med Assoc J 1992;146:473-81.

21 Owens DK. Interpretation of cost-effectiveness analyses.J Gen Intern Med 1998;13:716-7.

22 AIDS from maternally transmitted HIV Infection. J Med Screening 1997;4:177.

23 Centers for Disease Control. Public Health Service task force recommendations for the use of antiretroviral drugs in pregnant women infected with HIV-1 for maternal health and for reducing perinatal HIV-1 transmission in the United States. MMWR Morb Mortal Wkly Rep 1998; 47(RR-2):1-30.

24 Wade N, Birkhead GS, Warren BL, Charbonneau TT, French PT, Wang L, et al. Abbreviated regimens of zidovudine prophylaxis and perinatal transmission of the human immunodeficiency virus. $N$ Engl J Med 1998;339:1409-14.

25 McIntosh K. Short (and shorter) courses of zidovudine. N Engl J Med 1998;339:1467-8.

26 Mandelbrot L, Le Chenadec J, Berrebi A, Bongam A, Béniflor JL, Delfraissy JL, et al. Perinatal HIV-1 transmission: interaction between zidovudine prophylaxis and mode of delivery in the French perinatal cohort. JAMA 1998;280:55-60.

27 Intercollegiate Working Party for Enhancing Voluntary Confidential HIV Screening in Pregnancy. Reducing mother-to-child transmission of HIV infection in the UK. London: Royal College of Paediatrics and Child Health, 1998.

(Accepted 24 February 1998)

\section{Antenatal HIV testing: assessment of a routine voluntary approach}

Wendy M Simpson, Frank D Johnstone, David J Goldberg, Siobhan M Gormley, Graham J Hart

Papers pp 1650 ,

1656

Correspondence to:

Dr W M Simpson,

22 Lumsden Park,

Cupar, Fife KY15

$5 \mathrm{YL}$

wendy@lumsdenpark.

freeserve.co.uk

continued over

BMJ 1999;318:1660-1
The benefits of testing pregnant women for HIV are increasingly assured, particularly with regard to reducing vertical transmission. ${ }^{1}$ Yet uptake of antenatal HIV testing in Britain remains low. ${ }^{2}$ Our previous study examined an opt-in approach (women had to make an active choice to be tested). ${ }^{3}$ Some women were uncomfortable with this, feeling that it indicated high risk behaviour. We therefore assessed an approach based on similar requirements for information and consent but with a change in emphasis, in that testing was routine unless the woman declined.

\section{Subjects, methods, and results}

The testing programme was conducted during February to April 1998. Before their booking appointment, all women were sent a leaflet about blood tests to be conducted, including HIV testing. At the antenatal 